\title{
PANORÁMICA INSTITUCIONAL DEL PROCESO DE EVALUACIÓN EN LA ENSEÑANZA Y APRENDIZAJE ELECTRÓNICO
}

\author{
(INSTITUTIONAL ENVIRONMENT OF THE ASSESSMENT PROCESS IN E-LEARNING SYSTEMS)
}

Roberto Barchino Plata

Universidad de Alcalá (España)

\section{RESUMEN}

No cabe la menor duda, que la evaluación es una de las piezas clave en cualquier entorno de enseñanza y aprendizaje. Además, las actividades relacionadas con el proceso de evaluación en sistemas e-learning deben asociarse, obligatoriamente, con las especificaciones y estándares existentes para conseguir mejorar la interoperabilidad, la eficiencia y la eficacia de la propia evaluación. En este trabajo se presentan una serie de instituciones que trabajan e investigan en este ámbito y que puede servir de apoyo a cualquier investigador que se inicia en este campo.

\begin{abstract}
The assessment is without question one of the keys in any teaching and learning environment. Besides, the activities related to the assessment process in e-learning systems must be associated with the specifications and existing standards to improve the interoperability, efficiency and efficacy of the assessment. In this work it is presented a group of institutions that work and investigate in this environment.
\end{abstract}

\section{Aspectos generales}

La implantación de las tecnologías de la información y comunicación (TIC) en nuestra sociedad es algo habitual. En el campo de la enseñanza se agrupa en lo que se ha denominado e-learning o aprendizaje electrónico, que no es más, que el uso de dichas tecnologías e internet para ayudar al proceso de aprendizaje (Reparaz, 2003). Uno de los elementos clave en el diseño de una acción formativa, es sin lugar a dudas la evaluación. Prueba de ello, es la siguiente Arquitectura denominada Learning Technology System Architecture - LTSA creada por el Learning Technology Standards Committee del IEEE (IEEE P1484.1/D9, 2001), donde se definen las 
entidades básicas de un sistema e-learning: el alumno, el profesor (tutor virtual), la distribución de contenidos y finalmente los mecanismos de evaluación. La evaluación del aprendizaje se puede definir como la comprobación del grado de asimilación de conocimientos y habilidades establecidas a priori, que tiene como requisito la recogida de información, para emitir un juicio de valor que toma cuerpo en una calificación final.

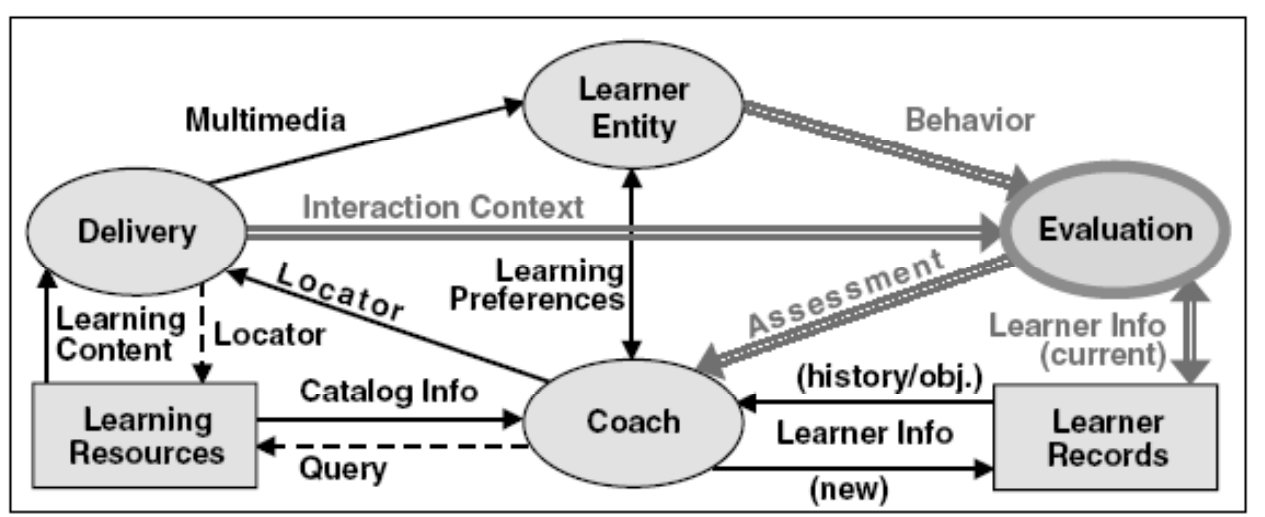

Fig. 1. Proceso de evaluación.

En la literatura anglosajona aparece el término Computer-Assisted Assessment CAA (Evaluación Asistida por Ordenador) donde se estudia la automatización del proceso de evaluación en distintas actividades, como por ejemplo, la presentación de preguntas, la corrección, la asignación de las calificaciones y por último la interpretación y análisis del examen. Por tanto, se puede afirmar que la evaluación asistida por ordenador no cuestiona el modelo de evaluación utilizado, sino que intenta, con la ayuda de las nuevas tecnologías, automatizar dicho proceso. Antes de planificar una estrategia de evaluación en la educación superior o en cualquier otro entorno, se deben tener en cuenta una serie de cuestiones fundamentales (McAlpine, 2002), como son el propósito de la evaluación, el aseguramiento de su validez y por último el diseño e implementación de pruebas con una calidad y un nivel de dificultad adecuadas.

Dentro del propósito de la evaluación se suele hablar de dos tipos: evaluación sumativa y formativa. La evaluación sumativa es la más conocida, en ella se comprueba los conocimientos adquiridos a través de distintas pruebas, emitiéndose juicios de valor - calificaciones. La evaluación formativa está integrada dentro del proceso 
ROBERTO BARCHINO

PANORÁMICA INSTITUCIONAL DEL PROCESO DE EVALUACIÓN EN LA ENSEÑANZA Y APRENDIZAJE ELECTRÓNICO

de aprendizaje y además se asocia a la utilidad de la evaluación como instrumento de mejora del proceso de enseñanza y aprendizaje, es decir, se establece una retroalimentación en dicho proceso para mejorar posibles deficiencias identificadas en la evaluación.

En 1956 Benjamin Bloom (Bloom, 1958) presentó una taxonomía que organiza, clasifica y ordena el aprendizaje y ayuda a diseñar cualquier actividad docente relacionada por ejemplo, con la evaluación. En concreto aparecen seis niveles de abstracción distintos. El primero es el Conocimiento, donde se dan las pautas para que el alumno memorice y recuerde cierta información concreta, el segundo es la Comprensión, donde el alumno debe entender y comprender lo aprendido, el tercer nivel es la Aplicación, para que el alumno utilice el conocimiento adquirido en nuevas situaciones, el cuarto es el Análisis, que se utiliza para que el alumno pueda dividir el conocimiento en partes y mostrar relaciones entre ellas, el quinto nivel es la Síntesis, donde el alumno será capaz de unir elementos del conocimiento adquirido para formar un todo y construir relaciones para nuevas situaciones y el último nivel es el de Evaluación, en el cual se deben establecer los juicios de valor a base de criterios previamente establecidos. A cada uno de los seis niveles presentados se les puede asociar técnicas de evaluación para comprobar si el alumno se sitúa en un nivel u otro.

Uno de los principales problemas detectados a la hora de investigar en el campo de la evaluación es, sin lugar a dudas, la ausencia de estándares universalmente aceptados. Aunque en la actualidad existen instituciones y organizaciones que están desarrollando estándares y especificaciones sobre tecnología educativa y en particular sobre la evaluación, éstos no están aceptados suficientemente. Existe una necesidad básica de estandarización que provea de los mecanismos necesarios para homogeneizar los estudios en este campo de trabajo. Por tanto, las investigaciones que se puedan realizar en este ámbito deben, obligatoriamente, venir de la mano de las recomendaciones existentes y si es necesario serán extendidas con nuevas y enriquecedoras ideas, con el fin último de convertirlas en estándares de facto, que toda la comunidad utilice. Esto es así debido a que la única institución que ha propuesto una auténtica y completa recomendación es IMS - Instructional Management System - con la especificación QTI - Question and Test Interoperability (Question and Test Interoperability Specification, 2006), que aunque abarca muchos temas, se centra en la interoperabilidad de exámenes entre sistemas distintos codificados sobre el lenguaje XML. Otras especificaciones que tienen relación con la evaluación y de la misma institución son: IMS Learning Design, IMS ePorfolio y IMS Learner Information Package. 
ROBERTO BARCHINO

PANORÁMICA INSTITUCIONAL DEL PROCESO DE EVALUACIÓN EN LA ENSEÑANZA Y APRENDIZAJE ELECTRÓNICO

Además del problema de la estandarización, en la actualidad están apareciendo nuevas formas y técnicas de evaluación que integran estrategias para valorar características, habilidades y competencias de los alumnos. Con estas nuevas formas de evaluación no es suficiente hacer un examen y evaluación acabada, sino que es necesario un trabajo muy intenso de investigación y desarrollo para estos novedosos mecanismos de evaluación.

\section{Instituciones en el ámbito de la evaluación}

En la actualidad existen multitud de organizaciones e instituciones que colaboran en la realización de recomendaciones y estándares en el campo de los sistemas e-learning. Algunas de ellas de enorme prestigio como Learning Technologies Standarization Committee de IEEE o 36 Subcommittee of the First joint International Standardization Organization and International Electrotechnical Commission Committee ISO/IEC JTC1 SC36. Para ofrecer una relación actualizada de instituciones, se ha utilizado el listado que mantiene el Observatorio de Estándares sobre Tecnologías de Formación, del Comité Europeo de Estandarización (European Committee for Standarization, 2006).

- ISO/IEC JTC1 SC36 - http://jtc1sc36.org/

- IEEE LTSC - http://ieeeltsc.org/

- CEN/ISSS - European Committee for Standardization - Information Society Standardization System - http://www.cenorm.be/cenorm/ index.htm

- $\quad$ ADL-Advanced Distributed Learning - http://www.adlnet.gov/index. $\mathrm{cfm}$

- $\quad$ AICC - Aviation Industry CBT Committee - http://www.aicc.org/

- IMS - Global Learning Consortium, Inc - http://www.imsglobal.org/

- European Schoolnet - http://www.eun.org/portal/index.htm

- ARIADNE - Foundation for the European Knowledge Pool - http:// www.ariadne-eu.org/

- EdNA Online - Education Network Australia - http://www.edna.edu. au/edna/page1.html

- DCMI - Dublin Core Metadata Initiative - http://dublincore.org/

Dentro del ámbito español es de reciente creación, en AENOR (Asociación Española de Normalización), el subcomité 36 "Tecnologías de la información para el aprendizaje" dentro del Comité Técnico de Normalización 71 "Tecnologías de la Información". Uno de los objetivos de este subcomité técnico es el seguimiento de 
los documentos, normas, especificaciones, etc. de carácter internacional que puedan ser adoptados a nivel nacional para así promover su uso.

En concreto, se presentan cuatro instituciones que han aportado significativos trabajos en el proceso de evaluación en sistemas e-learning. Las organizaciones son:

- IMS - Global Learning Consortium, Inc. Con la especificación Question and Test Interoperability.

- JISC - Joint Information Committee System. Con el Modelo de Referencia FREMA - Framework Reference Model for Assessment.

- $\quad$ CETIS - Center for Educational Technology Interoperability. Con el grupo de especial interés sobre evaluación. Asessement $S I G$ - Special Interest Group.

- OUN - Open Universiteit Nederland. Con el Modelo Educacional para la Evaluación - Educational Model for Assessment.

\section{IMS - Instructional Management System - Global Learning Consortium Inc.}

El proyecto IMS surge en el año 1997 por EDUCASE ${ }^{1}$, como un consorcio de instituciones, organizaciones educativas gubernamentales y empresariales con el ánimo de definir especificaciones para las tecnologías de formación a distancia. El objetivo básico de IMS es desarrollar y promover la adopción de especificaciones técnicas para la tecnología e-learning, centradas en la búsqueda de la interoperabilidad que permita que contenidos y entornos de aprendizaje distintos puedan convivir juntos. De hecho, varias especificaciones de IMS han llegado a convertirse en estándares de facto por su gran relevancia. Las especificaciones de IMS y publicaciones relacionadas se hacen públicas sin ningún coste adicional. Al día de hoy, IMS define y desarrolla sus especificaciones basadas en la interoperabilidad, gracias a la utilización de lenguaje XML. A través del lenguaje XML es posible el intercambio de contenidos, información sobre alumnos, evaluaciones, etc. entre distintos sistemas gestores de aprendizaje.

\section{Question and Test Interoperability Specification}

La especificación IMS Question and Test Interoperability (QTI) describe un modelo de datos para la representación de preguntas y exámenes y por supuesto sus 
correspondientes informes de los resultados. Además, QTI, permite el intercambio de un ítem - pregunta, examen o test y los resultados entre distintas herramientas de gestión del aprendizaje, repositorios, sistemas de evaluación, etc. El modelo de datos está descrito utilizando el lenguaje de notación UML - Unified Modeling Language - para facilitar la posterior implementación con cualquier herramienta o lenguaje de programación, sin embargo, para especificar el intercambio entre sistemas se ha utilizado el lenguaje XML - eXtensible Markup Language -. La actual versión de la especificación QTI es la 2.1 de enero de este año y se encuentra en estado de borrador público para que cualquier persona pueda sugerir cambios antes que se convierta en versión final.

A continuación se enumeran y definen una serie de beneficios que se desprenden del uso de esta especificación:

Mejora la interoperabilidad. La especificación QTI define un formato para compartir preguntas y exámenes entre sistemas.

Múltiples formatos de presentación. La presentación final de los test se puede realizar en distintos formatos.

Amplio rango de tipos de preguntas. La especificación QTI soporta selección múltiple, respuesta corta, escalas, completar frases, respuestas múltiples, etc...

Ahorro de tiempo y dinero. Se pueden diseñar planes de evaluación de una forma más rápida y sin ningún coste adicional a través de la especificación QTI, que además está muy extendida y aceptada.

Integración con otros entornos de trabajo. Se pueden incorporar los ítems QTI a otras especificaciones del sector de la capacitación y educación, tales como SIF (Schools Interoperability Framework) o SCORM - Modelo de referencia para objetos de aprendizaje distribuidos, del Departamento de Defensa de los EEUU.

Como se ha comentado la especificación QTI está diseñada para facilitar la interoperabilidad entre diferentes sistemas de gestión del aprendizaje. Específicamente, QTI está diseñado para proporcionar un formato adecuado y conocido para almacenar e intercambiar preguntas independientemente de la herramienta utilizada en la creación, soportar el desarrollo de repositorios a través de una gran variedad de tipos de aprendizajes y evaluaciones, proporcionar sistemas con la capacidad de informar de los resultados obtenidos en un examen de manera coherente. 
La siguiente figura muestra como está organizada una evaluación implementada a través de QTI, también se lista un pequeño ejemplo de codificación de una pregunta en formato XML-QTI.

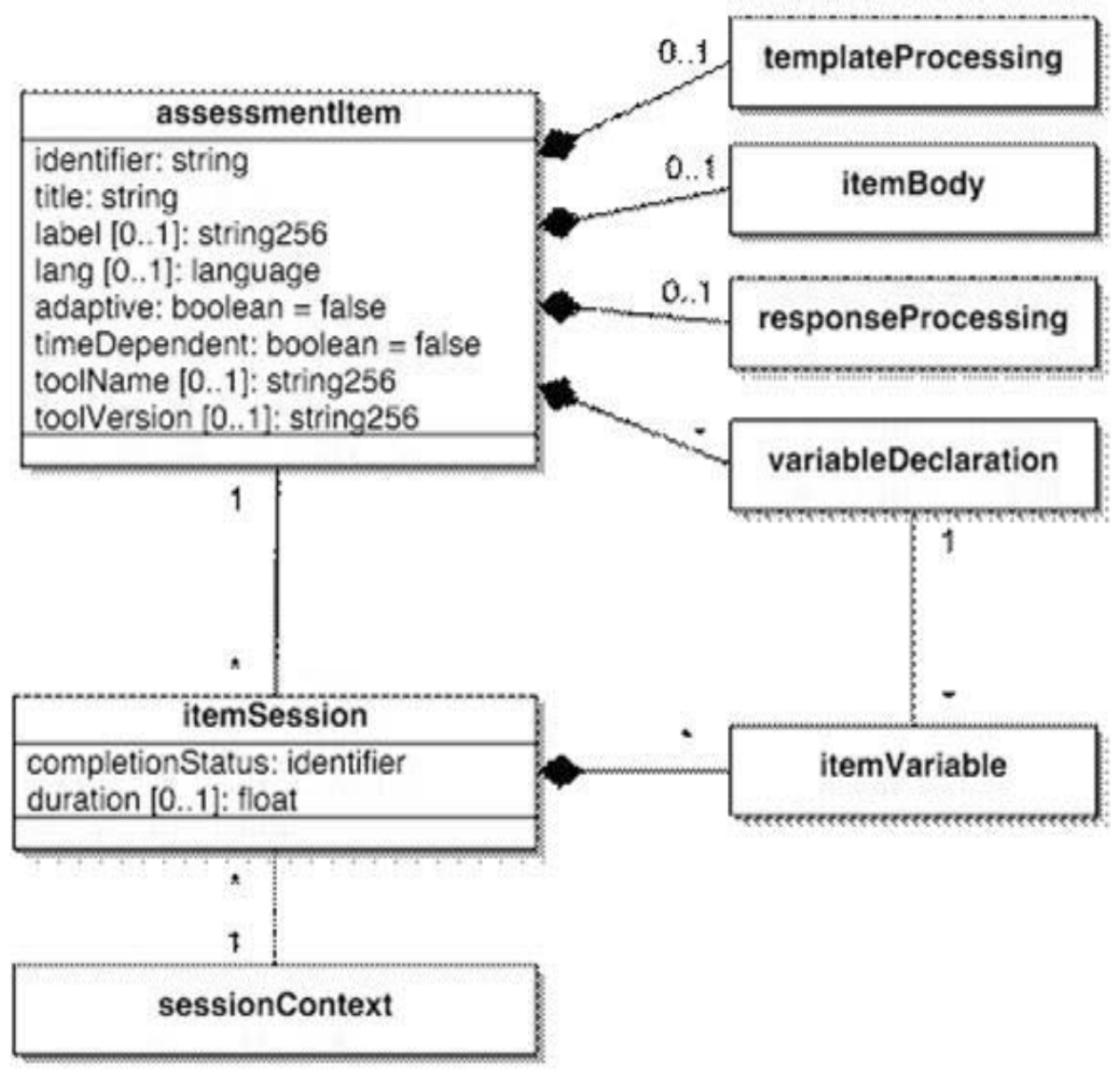

Fig. 2. Estructura de diseño de una evaluación QTI.

Por tanto, un assessmentItem es una composición de distintos elementos como se observa en la figura 2. En el siguiente código se puede ver como se implementa el diseño de una pregunta real utilizando para ello el lenguaje XML en formato QTI. 


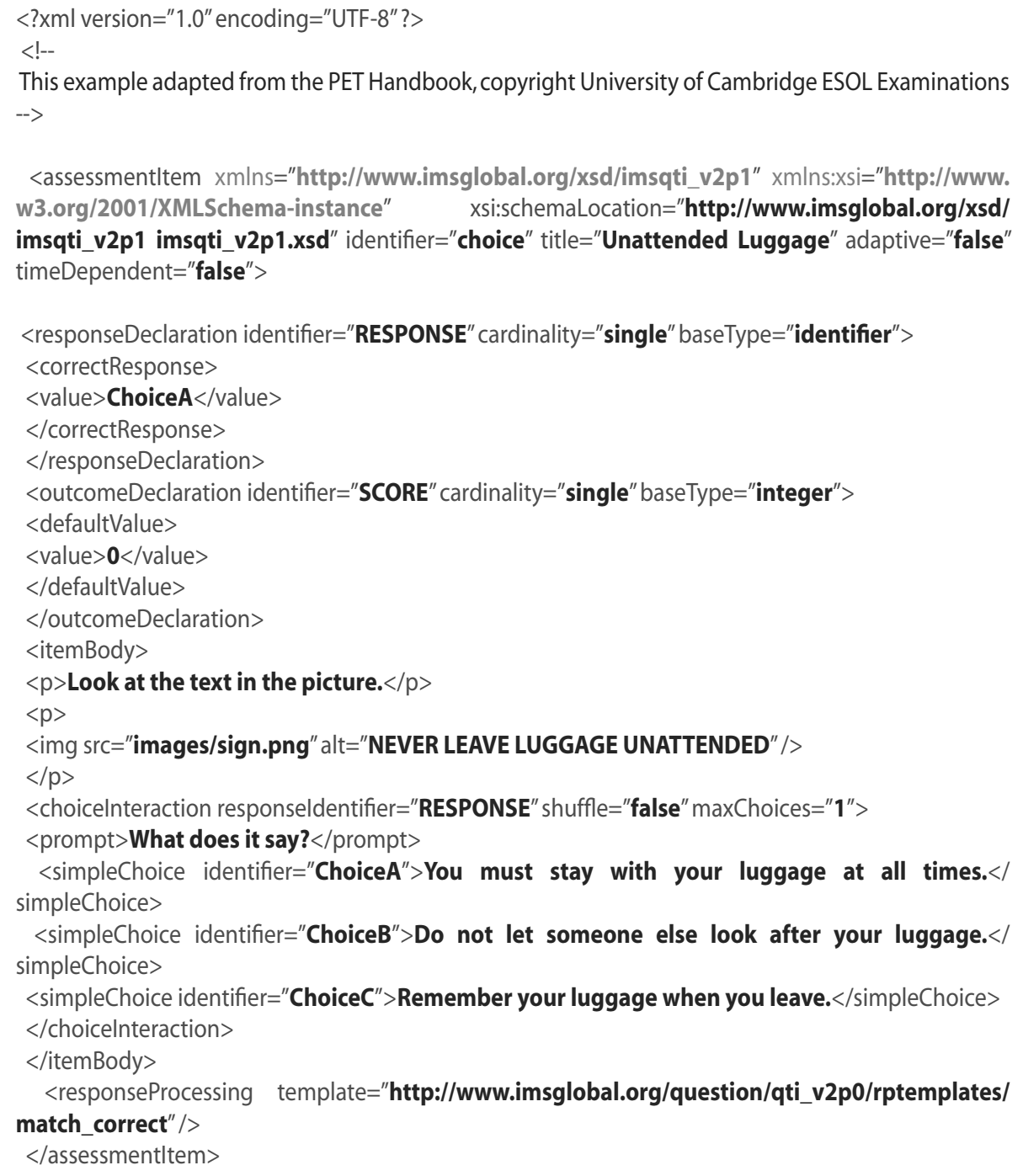

Fig. 3. Codificación pregunta QTI.

Evidentemente, debe existir un sistema que interprete adecuadamente el código anterior, para así poder visualizarlo como vemos en la siguiente figura. Así cualquier Sistema Gestor del Aprendizaje podrá, si cumple con la especificación, presentar y procesar de igual manera la pregunta. 


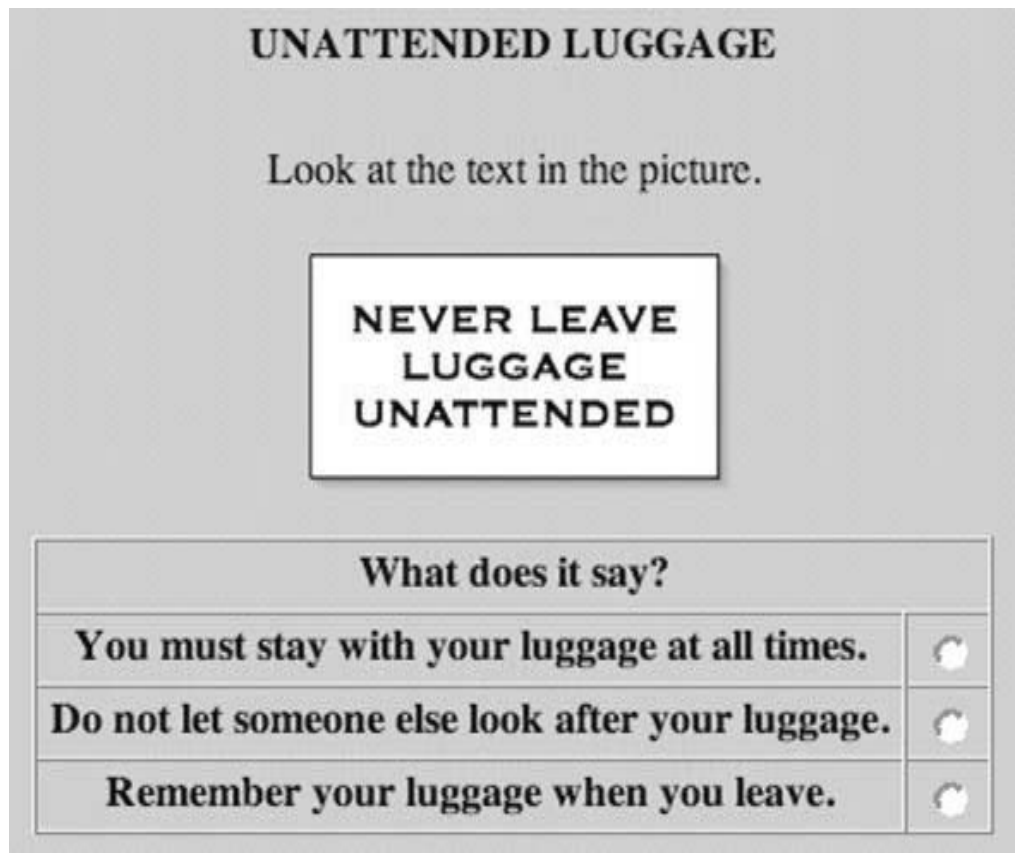

Fig. 4. Presentación pregunta QTI.

\section{JISC - Joint Information Committee Systems}

La institución JISC - Joint Information Systems Committee -, creada el 1 de Abril de 1993, es responsable del desarrollo y apoyo del e-learning en la educación superior del Reino Unido. Trabaja en la promoción y desarrollo de servicios relacionados con el uso de las tecnologías de la información en el ámbito de la educación universitaria y la investigación. Es un comité consultivo estratégico que colabora con distintas agencias financiadoras de la educación superior en Inglaterra, Gales e Irlanda del Norte, y promueve proyectos innovadores en la educación. Su objetivo es desarrollar políticas y programas estratégicos de alta calidad en el ámbito del e-learning. Entre los distintos proyectos que esta institución coordina, destaca por la relación con el tema a estudio, el proyecto FREMA (Framework Reference Model for Assessment).

\section{FREMA - Framework Reference Model for Assessment}

El proyecto FREMA forma parte del programa estratégico sobre e-learning del JISC (e-learning Framework) que tiene como principal objetivo identificar como 
los sistemas e-learning pueden facilitar el aprendizaje y aconsejar como éstos deben ser implementados. El objetivo de FREMA es desarrollar un modelo de referencia para sistemas en el entorno de la evaluación (e-assessment), construido sobre una arquitectura orientada a servicios, tales como los servicios web. El Modelo de Referencia sobre la Evaluación describe el proceso completo de diseño e implementación de una evaluación coherente y eficaz.

\section{Descripción del proyecto}

El proyecto establece una colaboración entre las Universidades inglesas de Southampton, Strathclyde y Hull, además el equipo investigador dispone de una serie de expertos en este dominio. Los miembros del equipo del proyecto FREMA han estado trabajando previamente en la definición e implementación de componentes de evaluación en iniciativas tales como:

- TOIA (Technologies for Interoperable Assessment) University of Strathclyde. http://www.toia.ac.uk/

- APIS (Assessment Provision through Interoperable Segments) University of Strathclyde.

- ASSIS (Assessment Sequencing) University of Hull. http://www.jisc. ac.uk/deletassis.html

- QTI SIG CETIS Question and Test Interoperability Special Interest Group. http://www.cetis.ac.uk/

En el proyecto FREMA se identifican los siguientes objetivos básicos:

- Definir los términos en el ámbito de la evaluación.

- Definir los patrones comunes para obtener una solución en el proceso de evaluación en términos de casos de uso y escenarios de trabajo.

- Proporcionar prototipos de los servicios del dominio de la evaluación, como prueba de la consistencia del Modelo de Referencia de Evaluación y permitir que el modelo sea evaluado.

En la siguiente figura se puede visualizar un resumen de las tareas o funciones más importantes en el dominio de evaluación. El proyecto FREMA se puede utilizar para encontrar gran cantidad de información útil en este entorno de trabajo, como son: estándares, proyectos de investigación relacionados, aplicaciones, especificaciones, guías, etc. 
ROBERTO BARCHINO

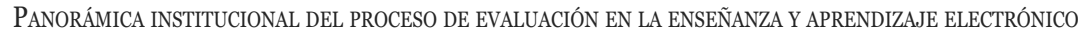

\section{Mapas Conceptuales}

Para conocer en detalle los puntos clave de este proyecto se deben presentar los mapas conceptuales que agrupan las entidades y los procesos de la evaluación. A continuación se presentan los dos mapas conceptuales: Mapa Conceptual de Entidades y Mapa Conceptual de Procesos. Estos mapas conceptuales agrupan todos y cada uno de los elementos de este modelo de referencia de evaluación.

\section{Mapas Conceptuales de Entidades}

Las entidades son los conceptos o términos que se manejan en este entorno y deben estar perfectamente definidos. La siguiente lista enumera los que FREMA propone.

Activity. Las técnicas utilizadas para evaluar candidatos. Se relacionan los diferentes tipos de preguntas - ítems.

Data. Información requerida para, producida para o relativa al proceso de evaluación.

Delivery Context. El entorno en el cual los candidatos son evaluados. Como por ejemplo un sistema informático.

Infrastructure. Sistemas de información de organizaciones, los cuales tienen alguna relevancia en el desarrollo de la evaluación.

Interaction type (IMS QTI). Tipos de interacciones permitidas a los candidatos para responder a las preguntas, seleccionando o construyendo la respuesta.

Pedagogical Context. La motivación en la educación: la evaluación.

Reports. Informes generados desde el procesamiento adecuado de la información.

Role. Las funciones asociadas a un usuario de un sistema de evaluación.

Specification. La legislación, estándares, guías y recomendaciones relacionadas con la evaluación.

Stakeholder. Aportaciones individuales y organizativas dentro del proceso de evaluación. 


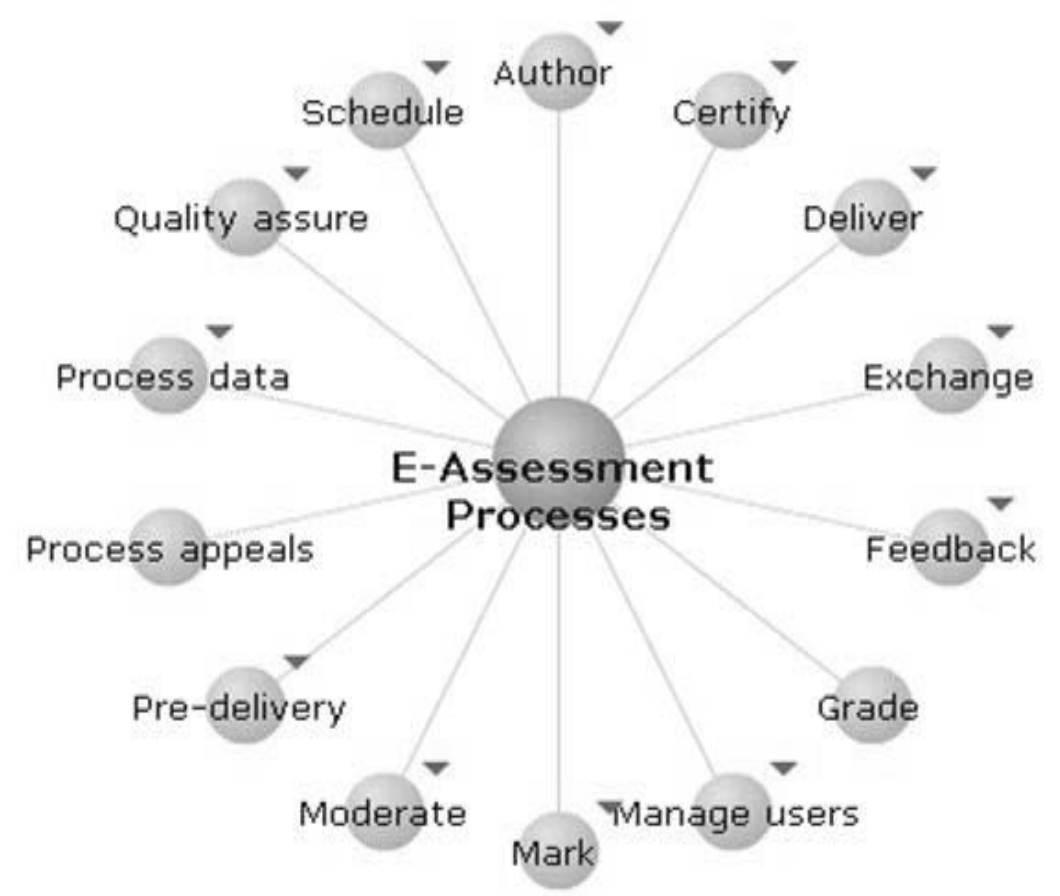

Fig. 5. Entidades de Evaluación.

\section{Mapas Conceptuales de Procesos.}

Este apartado presenta la definición de los procesos de evaluación más importantes del modelo FREMA.

Author. Será el creador de preguntas, almacenes, exámenes y plantillas.

Certify. Permite almacenar los resultados de un candidato.

Deliver. Presentación de una pregunta a un candidato.

Exchange. Permite el intercambio o compra de preguntas de un repositorio.

Feedback. Información presentada a un candidato para completar adecuadamente una pregunta o un examen.

Grade. Asignar a cada nivel de conocimiento una calificación. 
Manage Users. Establecer los usuarios del sistema, visualizando y eliminando, si así se desea, sus datos.

Mark. El proceso necesario para asignar una calificación a la respuesta de un candidato.

Moderate. Comprobar que los procesos de evaluación son satisfactorios.

Pre-delivery. Desarrollar con adelanto, las operaciones necesarias una vez que el examen tenga autor y horario asignado.

Process Appeals. Permitir a los candidatos que revisen la calificación obtenida.

Process Data. Generar un informe resumen de todos los exámenes realizados por el candidato.

Quality Assure. Llevar a cabo los procesos necesarios para asegurar que el material y el proceso de evaluación sean satisfactorios.

Schedule. Llevar a cabo las operaciones necesarias para preparar un examen.

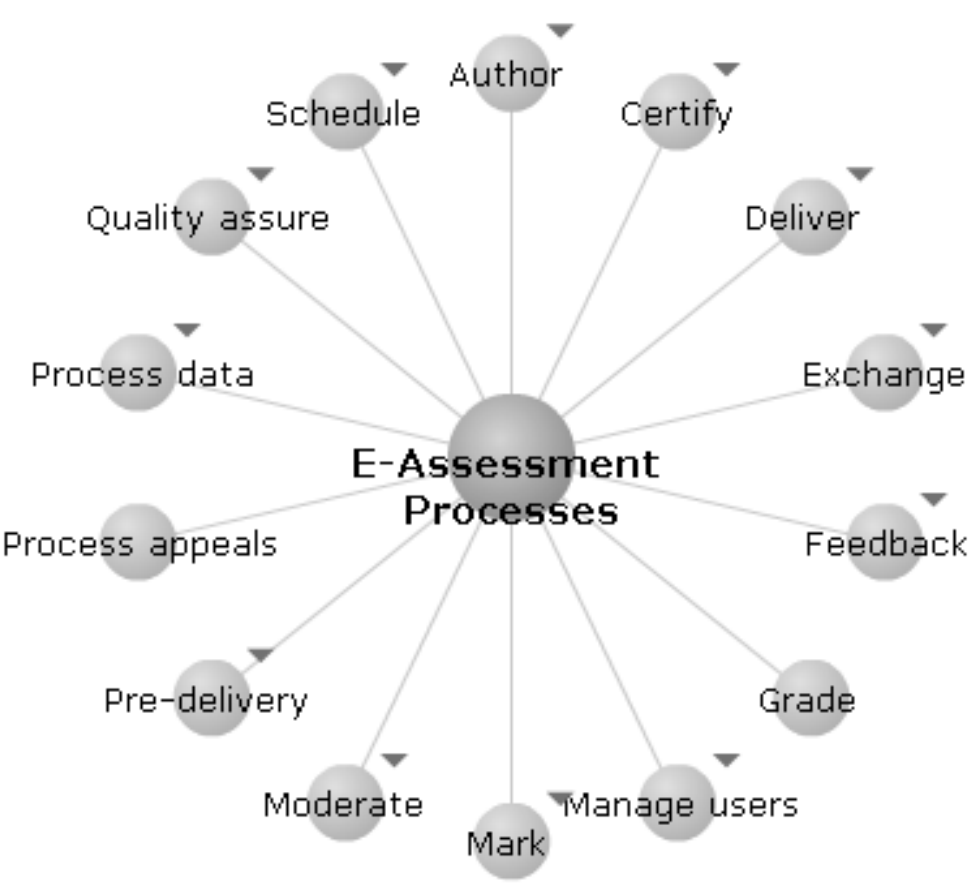

Fig. 6. Procesos de evaluación. 
La idea, por tanto, del modelo FREMA es aglutinar todos los esfuerzos y trabajos existentes para ser compartidos con cualquier institución que desee trabajar e investigar en este ámbito. Propone un entorno de trabajo a partir del cual se pueden implementar nuevos sistemas de evaluación de alta calidad.

\section{CETIS - Centre for Educational Technology Interoperability}

El CETIS representa a la Educación Superior inglesa en distintas iniciativas internacionales como:

- IMS Global Learning Consortium, que trabaja en la creación de especificaciones para lograr la interoperabilidad dentro de las tecnologías para el aprendizaje: metadatos, gestión de contenidos, accesibilidad, preguntas y exámenes.

- CEN/ISSS, Centro Europeo de Estandarización que desarrolla programas de trabajo para los estándares de las tecnologías educativas.

- IEEE Learning Technology Standards Committee, Comité de tecnologías de aprendizaje del IEEE.

La institución CETIS aconseja a Universidades y Escuelas sobre las estrategias técnicas y pedagógicas en la implantación de los estándares de las tecnologías de la educación. CETIS coordina grupos de especial interés -Special Interest Group: SIG-, sobre los siguientes temas:

- Metadata

- Education Content

- Question and Test

- Enterprise

- Accessibility

- ePortfolio

La institución CETIS está fundada por el JISC, Joint Information Systems Committee, y está dirigida por la Universidad anglosajona de Bolton. El CETIS, como se ha comentado anteriormente, coordina una serie de grupos de especial interés, esta iniciativa es una de las más interesantes ya que aglutina una serie de expertos en cada área de trabajo, investigando nuevas y prácticas políticas y planes de actuación relativos básicamente a los estándares actuales. 


\section{Assessment SIG - Special Interest Group}

El proceso de evaluación en la institución CETIS se estudia dentro del Grupo de Especial Interés sobre evaluación (ASIG). Este grupo ha sido establecido para controlar las iniciativas CAA basadas en la interoperabilidad dentro de la educación superior en el Reino Unido. El grupo de trabajo distribuye entre sus miembros toda la información relativa a las actividades para el estudio de los estándares y especificaciones además de proporcionarles un foro para el debate que permita el intercambio del conocimiento y de la experiencia entre sus miembros. Este grupo está coordinado por el departamento Learning Services de la Universidad de Strathclyde en Glasgow Inglaterra. El grupo tiene reuniones trimestrales donde se proporcionan a los participantes la oportunidad de aprender sobre las novedades aparecidas en el campo de la evaluación. Existe, también, un foro de discusión JISCMAIL, donde se puede unir cualquier persona para mantenerse informado sobre las acciones del grupo. El coordinador de este SIG es Rowin Young profesor de la Universidad de Strathclyde.

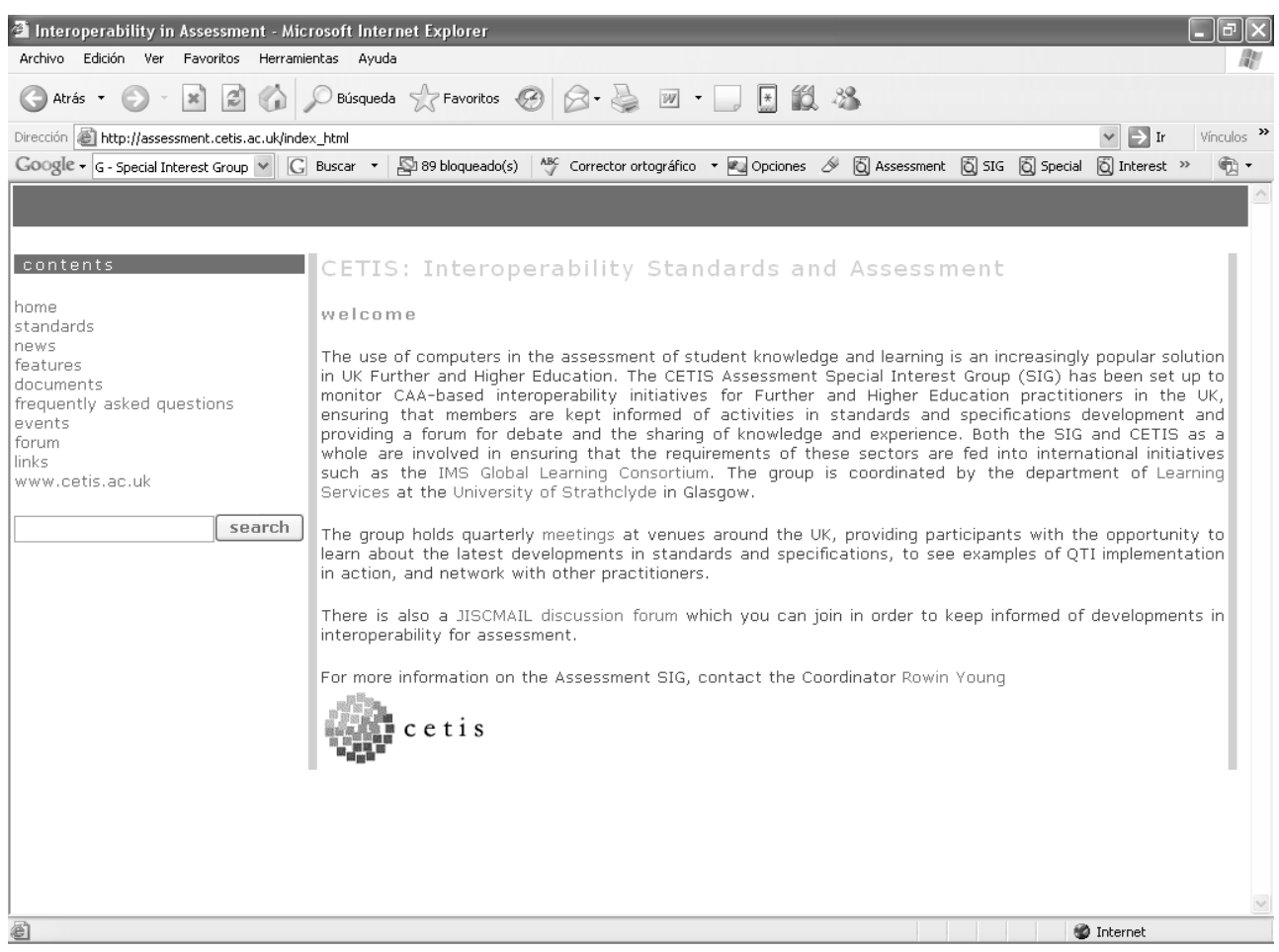

Fig. 7. Página principal del Assessment SIG. 


\section{OUN - Open Universiteit Nederland}

La Open Universiteit Nederland es una universidad a distancia como la Universidad de Educación Nacional a Distancia - UNED - en España. Esta universidad trabaja de manera muy nítida en la utilización de las nuevas tecnologías en el proceso de aprendizaje. El fruto más importante, sin lugar a dudas, es el lenguaje EML (Educational Modelling Language), que recientemente ha sido adoptado por IMS dentro de la especificación, para el diseño del aprendizaje, Learning Design. Además de este lenguaje, la universidad Holandesa ha propuesto un modelo educacional para la evaluación que se presenta en el siguiente apartado.

\section{Educational Model for Assessment}

La tendencia actual en la evaluación no está centrada en la estandarización de exámenes tipo test con preguntas de múltiples respuestas sino en la evaluación, más compleja, de las características, habilidades, perfiles específicos en un dominio de evaluación de candidatos.

También se están desarrollando nuevos tipos de evaluación, como es la evaluación de competencias. Por ello, se deben crear modelos conceptuales abiertos y que den soporte a nuevas formas de evaluación que pudieran aparecer.

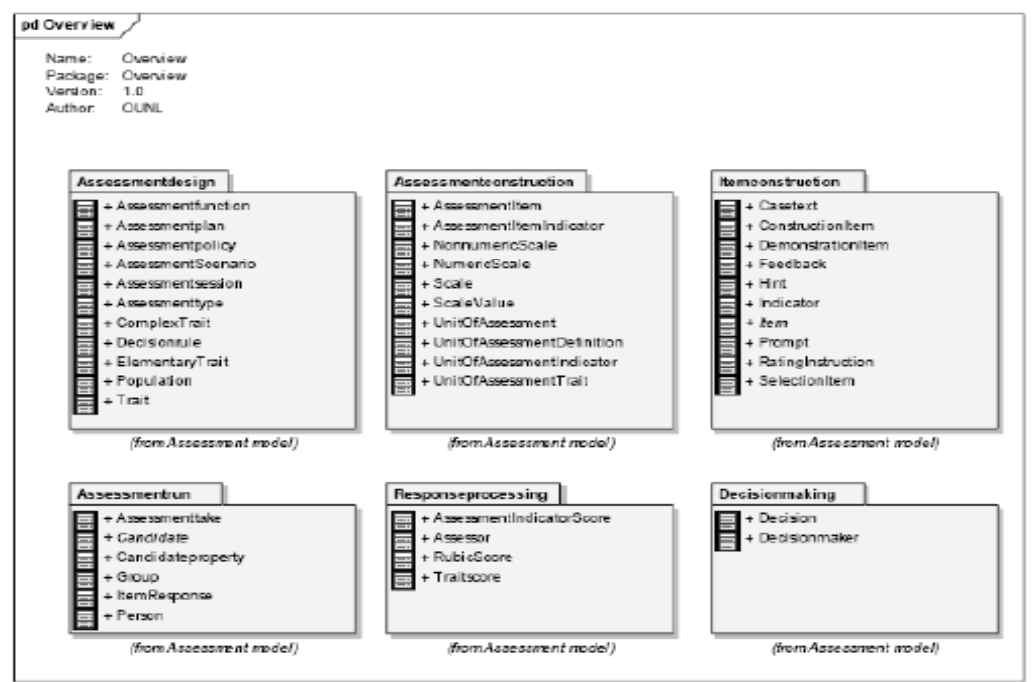

Fig. 8. Resumen de las clases del modelo. 
El modelo diseñado cumple una serie de requisitos, que se relacionan a continuación (Hermans, 2005):

1. Flexibilidad: El modelo de evaluación debe ser capaz de describir las evaluaciones basadas en diferentes teorías y modelos.

2. Formalización: El modelo de evaluación debe ser capaz de describir las evaluaciones y sus procesos de manera formal.

3. Reusabilidad: El modelo de evaluación debe hacer posible la identificación e intercambio de objetos (preguntas, exámenes, unidades de evaluación, competencias, planes de evaluación) y su reutilización en otros contextos.

4. Interoperabilidad y sostenibilidad: La separación entre los estándares y técnicas debe ser realizada, además el modelo de evaluación debe resistir los cambios tecnológicos.

5. Completitud: El modelo de evaluación debe cubrir el proceso entero de evaluación, incluyendo todos los tipos de objetos y sus relaciones y el flujo de trabajo.

6. Tipos de objetos: El modelo de evaluación debe ser capaz de poder expresar el significado semántico de los diferentes objetos dentro de un contexto de evaluación.

7. Repetición: El modelo de evaluación debe describir las evaluaciones para si es posible repetir la ejecución.

8. Neutralidad: La descripción de una evaluación, donde sea posible, debe ser neutral, por tanto ésta puede ser implementada en diferentes formatos: web, papel, test, etc.

9. Compatibilidad: El modelo de evaluación debe ser compatible con los estándares y especificaciones disponibles.

El Modelo Educacional para la Evaluación está diseñado mediante el lenguaje de modelado UML, además éste se encuentra dividido en varios sub-modelos, cada uno se centra en un nivel distinto del propio modelo de evaluación. A continuación se presenta una breve descripción de las clases más importantes del modelo y de las relaciones existentes entre ellas. 
Candidato: Un candidato es la persona o grupo que se va a evaluar. Es una clase abstracta y heredan de ella:

- Persona: cuando la evaluación es individual.

- Grupo: cuando se realiza para más de una persona.

Rasgo/Característica Cada grupo o persona tiene una serie de rasgos, que son los que se intentan evaluar.

- Indicadores: son los indicadores que nos definen el grado de acercamiento al rasgo.

- Regla de decisión: regla que determina el nivel de conocimiento sobre un rasgo o característica que tiene un candidato.

Población: Los candidatos pertenecen a una población, que tiene una serie de propiedades que los distinguen de otras poblaciones, por ejemplo tipo de escolarización.

Plan de Evaluación: Define como se debe reunir la información de un candidato que pertenece a una población, para tomar la decisión que se describe en función evaluación.

Política de evaluación: Determina las reglas que se han de seguir para la gestión del plan de evaluación. Ambos, política y plan, prescriben un tipo evaluación.

- Tipo de evaluación: determina si la evaluación va a ser un examen oficial o por contrario un ejercicio de entrenamiento.

Elemento: Es la menor unidad de medida para estimar el conocimiento de un candidato sobre una característica. Es una clase abstracta y heredan de ella:

- Elemento Selección: las respuestas se eligen de un grupo que se presenta (tipo test).

- Elemento Demostración: se observa al candidato implementando.

- Elemento Desarrollo: el candidato ha de proporcionar una respuesta corta.

Respuesta Elemento Respuesta realizada por el candidato al enunciado del elemento. 
- Calificador: El responsable de traducir las respuestas de un candidato en un código.

- Escala: Especificación de cómo la respuesta de un candidato se debe traducir en puntuación. Se tienen 2 tipos de escalas:

1. Escala numérica.

2. Escala No numérica.

Unidad de Evaluación Es la menor agrupación de elementos para presentar a uno o más candidatos destinados a evaluar un rasgo o característica.

- Definición de Unidad de Evaluación: se definen una serie de parámetros sobre la unidad de evaluación.

Escenario Evaluación Conjunto de reglas que determinan las unidades de evaluación que se van a distribuir a los candidatos, basadas en las propiedades de los mismos.

- Sesión Evaluación: Registra la fecha, hora de inicio y hora fin.

- Realizar Evaluación: Registra los datos del candidato y de las unidades de evaluación.

\section{CONCLUSIONES}

En este artículo se han presentado las instituciones y las aportaciones más importantes de éstas en el ámbito de la evaluación en la enseñanza y aprendizaje electrónico. El éxito de las acciones formativas virtuales dependen básicamente de unos contenidos docentes de calidad, además de establecer una serie de procesos encaminados en el establecimiento de tareas y actividades destinadas a la evaluación del alumno. Conocer cuales son las organizaciones más relevantes en el proceso de evaluación es básico si queremos conseguir cursos virtuales adecuados para calificar correctamente a un determinado perfil de alumno y sus competencias asociadas. 


\section{NOTAS}

$1 \quad$ EDUCASE es una asociación sin ánimo de lucro, cuya misión principal es la de avanzar en la educación superior con el uso inteligente de las tecnologías de la información, en EEUU.

\section{REFERENCIAS BIBLIOGRÁFICAS}

Benjamin S. Bloom (1958). Taxonomy of educational objectives, handbook 1 : Cognitive domain. England: Logman.

European Committee for Standarization (2006). Information Society Standartization System. Learning Technologies Standards Observatory. Disponible en: http://www.cenorm. be/cenorm/businessdomains/ businessdomains/isss/index.asp.

H. Hermans et al. (2005). Educational model for Assessment Version 1.o. Educational Technology Expertise Centre OTEC. Open Universitiet Nerderland.
IEEE P1484.1/D9, 2001. Draft Standard for Learning Technology - Learning Technology Systems Architecture (LTSA). Disponible en: http://edutool. com/ltsa/o9/IEEE 1484 o1 Do9 LTSA.doc

Mhari McAlpine (2002) Principles of assessment, CAA Centre, University of Luton., England.

Question and Test Interoperability Specification (2006). IMS Global Learning Consortium. Disponible en: http://www.imsglobal.org/question/ index.html

Reparaz et al. Mir (2003). La formación en Internet. Modelo de un curso online. Barcelona: Ariel.

\section{PALABRAS CLAVE}

E-learning, Evaluación, Instituciones, Especificación.

\section{KEYWORDS}

Assessment, specification, institutions. 
ROBERTO BARCHINO

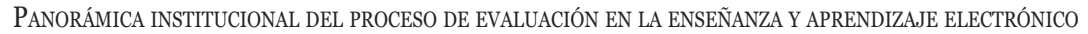

\section{PERFIL ACADÉMICO DEL AUTOR}

Roberto Barchino es Ingeniero en Informática por la Universidad Politécnica de Madrid. Profesor Colaborador del Área de Lenguajes y Sistemas Informáticos del Departamento de Ciencias de la Computación de la Universidad de Alcalá y Profesor Tutor de la Universidad Nacional de Educación a Distancia (UNED) en el Centro Asociado de Guadalajara. Es autor de más de 60 trabajos científicos (libros, artículos, proyectos/contratos de investigación, etc.), algunos de ellos relacionados directamente con la Tecnología Educativa. Además es vocal del Comité Técnico de Normalización 71, Subcomité 36 sobre Tecnologías de la Información para el Aprendizaje de AENOR Asociación Española de Normalización y Certificación.

Dirección postal: $\quad$ Dpto. de Ciencias de la Computación E.T.S. Ingeniería Informática. Universidad de Alcalá Campus Universitario. 28871. Alcalá de Henares - Madrid roberto.barchino@uah.es

Fecha de recepción: 11.07.06

Fecha de aceptación: 03.11.06 